\title{
General Flavour Blind MSSM and CP Violation
}

\author{
Alfred Bartl* \\ Institut für Theoretische Physik, Universität Wien, A-1090, Vienna, Austria \\ E-mail: bartīap.univie.ac.ati
}

\section{Thomas Gajdosik}

University of Alabama, Tuscaloosa, Alabama 35487, USA

E-mail: 'garfield@ghepu3.oeaw.ac.at'

\section{Enrico Lunghi}

Deutches Elektronen Synchrotron DESY, Hamburg

E-mail: 'iunghi@i@ail. desy.dé

\section{Antonio Masiero}

SISSA - ISAS and INFN, Sezione di Trieste, I-34013, Trieste, Italy

E-mail: masiero@he.sissa.it'

\section{Werner Porod}

Institut für Theoretische Physik, Universität Zürich, CH-8057, Zürich, Switzerland E-mail: iporod@̄physik. unizh.chi

\section{Hanns Stremnitzer}

Institut für Theoretische Physik, Universität Wien, A-1090, Vienna, Austria

E-mail: 'strem@ap.univie.ac.ati

\section{Oscar Vives}

Department of Physics, Theoretical Physics, U. of Oxford, Oxford OX1 3NP, UK

E-mail: 'vives@thphys.ox.ac.uki

ABstract: We study FCNC and $C P$ violating processes in the MSSM without a new flavour structure (flavour blind MSSM). The low energy parameters are determined by the running of the soft breaking terms from the GUT scale with SUSY phases consistent with the EDM constraints. We find that the $C P$ asymmetry in $b \rightarrow s \gamma$ can reach large values potentially measurable at $\mathrm{B}$ factories, especially in the low $B R(b \rightarrow s \gamma)$ region. We analyze the SUSY contributions to the anomalous magnetic moment of the muon pointing out its impact on the $b \rightarrow s \gamma C P$ asymmetry and on the SUSY spectrum.

\footnotetext{
${ }^{*}$ Speaker.
} 
In this contribution we study in a systematic way the restrictions on the supersymmetry (SUSY) parameters and complex phases which can be derived from the experimental information on flavour changing neutral currents (FCNC) and on $C P$ violation. We choose the Minimal Supersymmetric Standard Model (MSSM) as our theoretical framework and focus on a class of SUSY models that we call flavour blind MSSM. With this term we refer to a model where the soft breaking terms at the grand unification (GUT) scale do not introduce any new flavour structure beyond the usual Yukawa matrices. In this restricted class of models, the number of parameters is largely reduced and it is therefore possible to perform a complete phenomenological analysis. Here we present the main results of our analysis. For details we refer to [i]

We consider two cases, which are specified by the structure of the soft breaking parameters at the GUT scale. The first case is the simplest version of the constrained MSSM, where we take the following independent parameters:

(I) $M_{1 / 2}, M_{0}^{2},\left|A_{0}\right|, \phi_{A_{0}}, \phi_{\mu}$, and $\tan \beta$,

which are the universal gaugino and scalar mass parameter, the absolute value and the phase of the trilinear scalar coupling, the phase of $\mu$, and the ratio of the vacuum expectation values of the Higgs fields. The second case refers to the SUSY SU(5) model, where we take the following set of parameters:

(II) $M_{1 / 2}, M_{5}^{2}, M_{10}^{2}, M_{H_{1}}^{2}, M_{H_{2}}^{2},\left|A_{u}\right|,\left|A_{d}\right|, \phi_{A_{u}}, \phi_{A_{d}}, \phi_{\mu}, \tan \beta$, where now $M_{5}^{2}, M_{10}^{2}, M_{H_{1}}^{2}$ and $M_{H_{2}}^{2}$ are the scalar mass parameters of the $\overline{5}$ and 10 sfermions and the two Higgs doublets, and $A_{u}$ and $A_{d}$ are the trilinear scalar couplings of the 10 and $\overline{5}$.

With the SUSY parameters defined at the GUT scale we determine the soft SUSY breaking parameters at the weak scale by evolving them down with the renormalization group equations (RGE). In our analysis, we have used two-loop RGEs as given in [2]

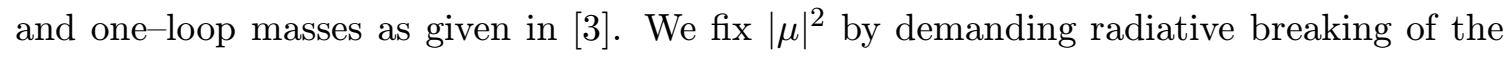
electroweak $S U(2)_{L} \times U(1)$ symmetry. At the weak scale we impose the constraints from direct SUSY and Higgs particle searches [4, and from the $\rho$-parameter, as well as the requirements of colour and electric charge conservation and the lightest SUSY particle (LSP) to be neutral. With these sets of soft SUSY parameters we calculate the electric dipole moment $(\mathrm{EDM})$ of the electron and the branching ratio of $b \rightarrow s \gamma$ and compare them with the experimental data, $\left|d^{e}\right| \leq 4.0 \times 10^{-27} e \mathrm{~cm}$ and $2 \times 10^{-4} \leq B R(b \rightarrow$ $s \gamma) \leq 4.5 \times 10^{-4}\left[\begin{array}{l}50 \\ {[5}\end{array}\right]$. The sets in agreement with the experimental constraints are used to calculate the $C P$ asymmetry of $b \rightarrow s \gamma$ and the SUSY contributions to $\epsilon_{K}, \Delta M_{B_{d}}$ and $\Delta_{M_{s}}$. We also calculate the SUSY contributions to the muon anomalous magnetic moment $a_{\mu^{+}}$in order to quantify the effect of the recent experimental data on the observables we are interested in. We assume that the SUSY contribution accounts for the difference

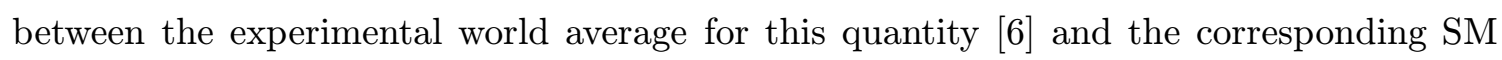

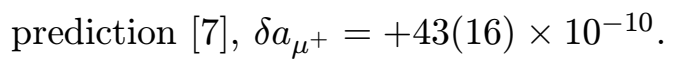


We first study the restrictions on the phases from the electron EDM constraint. Due to cancellations between different contributions these restrictions are less stringent and are very different from the case that the EDM constraint is required for each contribution separately (see [i] and gaugino masses at $M_{G U T}$ in the range $100 \mathrm{GeV}<M_{i}<1 \mathrm{TeV}$, the trilinear terms $0<\left|A_{d}\right|^{2}<M_{10}^{2}+M_{5}^{2}+M_{H_{1}}^{2}, 0<\left|A_{u}\right|^{2}<2 M_{10}^{2}+M_{H_{2}}^{2}$, taking their phases arbitrary, and $4<\tan \beta<50$. We find that the EDM constraint implies correlations between $\phi_{\mu}$ and $\phi_{A_{0}}$ and between $\phi_{\mu}$ and $M_{0}^{2}$. While it is possible to find any value for $\phi_{A_{0}}$, the values of $\phi_{\mu}$ are more constrained: $\phi_{\mu} \lesssim 0.1$ for lower values of $M_{0}^{2}$, whereas values up to $\phi_{\mu}=0.4$ are allowed for higher $M_{0}^{2}$.

Next we discuss the main features of the SUSY particle spectrum relevant for $C P$ violating and FCNC observables. In Fig. ${ }_{1}^{1} i_{1}$ we show the scatter plot of the lightest chargino versus the lightest stop masses for the SUSY $S U(5)$ model (set (II)). All points fulfill the $\mathrm{EDM}$ and $B R(b \rightarrow s \gamma)$ constraints, whereas the black dots are the points of the parameter space that also fulfill the $\delta a_{\mu^{+}}$constraint. We confirm the presence of an upper bound on the chargino mass of about $700 \mathrm{GeV}$ for very large $\tan \beta$ (of order 50) [9.9], and lower for smaller values of $\tan \beta$, which is a consequence of the $\delta a_{\mu^{+}}$constraint. It can be shown that this bound is essentially due to our assumption of gaugino mass unification. We would like to stress, however, that in any RGE evolved MSSM with gaugino mass unification the $a_{\mu^{+}}$constraint has important consequences on the complete MSSM spectrum. As can be seen in Fig. $i_{-1}^{-}$there is a strong correlation among the stop and chargino masses due to the dominant gaugino RGE effects. Then, without any further restriction on the SUSY parameter space, this correlation implies the presence of an upper bound on the light stop mass of $m_{\tilde{t}} \leq 1500 \mathrm{GeV}$.

An especially interesting observable is the $C P$ asymmetry in the partial width,

$$
A_{C P}^{b \rightarrow s \gamma}=\frac{B R\left(\bar{B} \rightarrow X_{s} \gamma\right)-B R\left(B \rightarrow X_{s} \gamma\right)}{B R\left(\bar{B} \rightarrow X_{s} \gamma\right)+B R\left(B \rightarrow X_{s} \gamma\right)}
$$

The SM prediction for this asymmetry is exceedingly small, therefore, it is sensitive to the new SUSY phases. As $\phi_{\mu}$ and $\phi_{A}$ are associated with chirality changing operators,

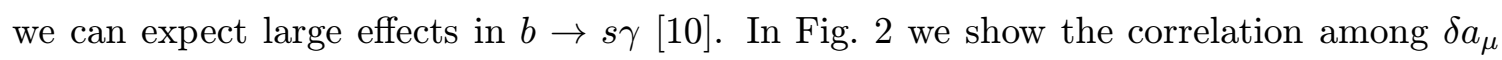
and $\operatorname{BR}(b \rightarrow s \gamma)$. All points shown fulfill the EDM constraint, whereas the black dots represent the points of the parameter space that also reproduce the measured anomalous magnetic moment. In this plot we can see that the $\delta a_{\mu}$ constraint cuts out the region of high branching ratios $B R(b \rightarrow s \gamma)$. In our flavour blind scenario the main contributions to the $b \rightarrow s \gamma$ decay are due to charged Higgs, light stop and light chargino loops. The $B R(b \rightarrow s \gamma)$ constraint and the $\delta a_{\mu}$ constraint favour $\operatorname{Re}(\mu)>0$. Imposing the EDM constraint reduces the $C P$ asymmetry to less than $1 \%$ in the region of large branching 
ratio $B R(b \rightarrow s \gamma)$. However, for small $B R(b \rightarrow s \gamma)$ the $C P$ asymmetry can go up to $5 \%$, making it potentially measurable at $B$ factories.

Finally we calculated the SUSY contributions to the $C P$ violating quantities $\epsilon_{K}$, $\Delta M_{B_{d}}, \Delta M_{B_{s}}$ and studied the modifications of the unitarity triangle. We obtain only small deviations from the SM predictions. This result is different from those obtained in the context of Minimal Flavour Violation MSSM or in the MSSM with non-universal parameters [i]

Acknowledgements: This work was supported by the 'Fonds zur Förderung der wissenschaftlichen Forschung' of Austria, FWF Project No. P13139-PHY, by the EU TMR Network Contracts No. HPRN-CT-2000-00148, HPRN-CT-2000-00149 and HPRN-CT2000-00152 and by Cooperazione scientifica e tecnologica Italia-Austria 1999-2000, Project No. 2; W.P. was supported by the Spanish Ministry of Education and Culture under the contract SB97-BU0475382 and by DGICYT grant PB98-0693; T.G. acknowledges financial support from the DOE grant DE-FG02-96ER40967; E.L. and O.V. thank SISSA for support during the first stages of this work. E.L. was supported by the Alexander Von Humboldt Foundation; O.V. acknowledges financial support from a Marie Curie E.C. grant (HPMFCT-2000-00457) and partial support from Spanish CICYT AEN-99/0692.

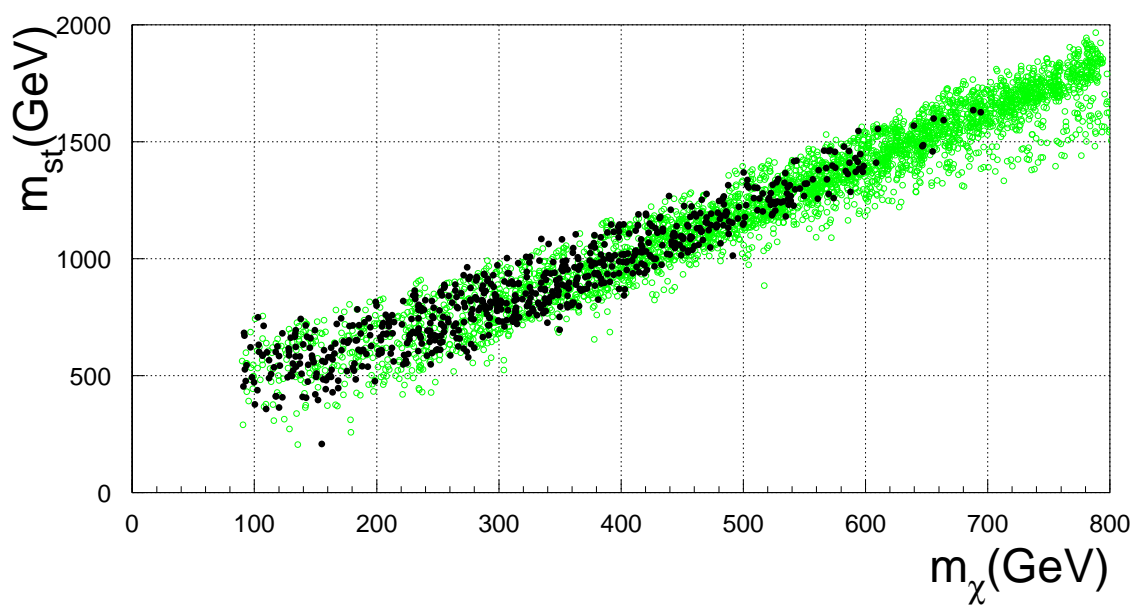

Figure 1: Chargino-stop mass correlations in the $S U(5)$ inspired MSSM with $b \rightarrow s \gamma$ constraint satisfied. The black dots satisfy also the $a_{\mu^{+}}$constaint.

\section{References}

[1] A. Bartl, T. Gajdosik, E. Lunghi, A. Masiero, W. Porod, H. Stremnitzer, O. Vives, Phys. Rev. D 64, 076009 (2001).

[2] S. P. Martin and M. T. Vaughn, Phys. Rev. D 50, 2282 (1994).

[3] D. M. Pierce, J. A. Bagger, K. Matchev and R. Zhang, Nucl. Phys. B 491, 3 (1997). 


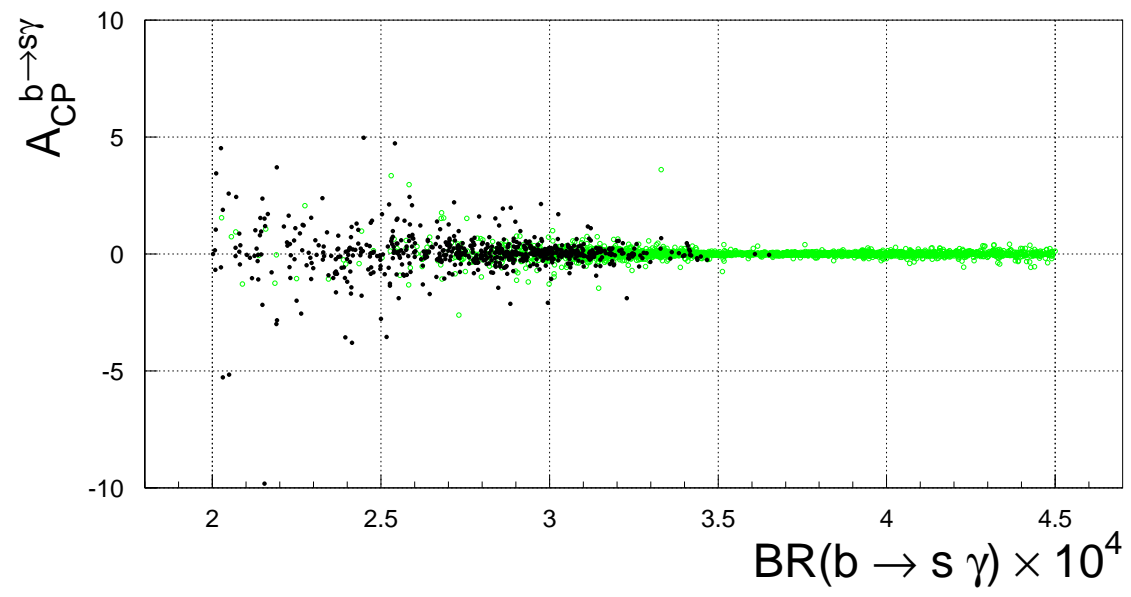

Figure 2: Plot of the $C P$ asymmetry versus the branching ratio of $b \rightarrow s \gamma$. We allow only for points whose phases satisfy the EDM's constraints. The black dots satisfy the $a_{\mu^{+}}$constraint.

[4] D. E. Groom et al. [Particle Data Group Collaboration], Eur. Phys. J. C 15, 1 (2000).

[5] S. Ahmed et al. (CLEO Collaboration), CLEO CONF 99-10, hep-ex/9908022; K. Abe et al. [Belle Collaboration], hep-ex/0103042.

[6] H. N. Brown et al. [Muon g-2 Collaboration], hep-ex/0102017.

[7] A. Czarnecki and W. J. Marciano, hep-ph/0102122.

[8] A. Bartl, T. Gajdosik, W. Porod, P. Stockinger and H. Stremnitzer, Phys. Rev. D 60, 073003 (1999)

[9] J. L. Feng and K. T. Matchev, hep-ph/0102146.

[10] D. A. Demir, A. Masiero and O. Vives, Phys. Lett. B479, 230 (2000); Phys. Rev. D61, 075009 (2000).

[11] A. Ali and D. London, Eur. Phys. J. C 9, 687 (1999); A. J. Buras, P. Gambino, M. Gorbahn, S. Jager, L. Silvestrini, Phys. Lett. B 500, 161 (2001); A. J. Buras and R. Buras, Phys. Lett. B 501, 223 (2001).

[12] E. Lunghi, these Proceeding; O. Vives, these Proceedings 